\title{
Using Concatenated Steganography for Visual Analysis in GIS SOA
}

\author{
Andrew Yershov ${ }^{1}$, Vitaly Zabiniako ${ }^{2}$, Pavel Semenchuk ${ }^{3}{ }^{1-3}$ Riga Technical University
}

\begin{abstract}
Geographic Information Systems (GIS) perform the computational processing of geographic data and store the geometry and the attributes of data that are geo-referenced, that is, situated on the earth surface and represented in a cartographic projection. Integral part of GIS functionality is an opportunity to make a complex analysis, which is able to integrate geo-referenced imagery as data layers or themes and link them to other data sets producing geospatial renderings of data. Based on Service-Oriented Architecture (SOA) GIS expose its capabilities as Web services with purpose to create a flexible and extensible GIS that can quickly respond to changing and future organizational needs. In that case client's application sends specially formed requests to the GIS services and ensures visual processing of responses. But the drawback is the count of such requests, because imaginary data and data needed for analysis are received sequentially (not simultaneously). The purpose of this paper is to present the solution of decreasing the amount of geo-requests, enabling extensions to existing GIS visual analysis tools and bringing into GIS SOA synchronization mechanism designed on principles of concatenated steganography. This ensures that various geospatial data streams will be kept, transmitted and analytically processed together using concatenated steganography, strongly taking into account mutual relations between these streams.
\end{abstract}

Keywords - Visual analysis, concatenated steganography, GIS SOA, Web Map Service.

\section{INTRODUCTION}

There are many existing multi-purpose services, available through the Internet. Nevertheless, while working separately, these services cannot support the complicated processes that are required, to ensure the satisfaction of needs of different users. These services have to communicate with each other, to enable according necessary complicated processes for users. The Service-Oriented Architecture (SOA) ensures the possibility to manage separate Web services in such a way that they cooperate with each other while organizing the complicated business processes and meeting the demands of users for multiform information systems.

By applying SOA, it is possible to implement and manage the geographic information system (GIS). By using the Internet it is possible for users to view the geographic objects, which are depicted in high quality. This is ensured by special services - the services for receiving geographic data and the services for visualization of this data. To exploit GIS and advantages of these objects fully, it is necessary to keep data about geographic objects in the united, publicly available repository (for example - data base). It is also important, that the applications, that use these data, could cooperate with each other. Without respect to these demands it is quite difficult to use effectively the geospatial data, services and processes, to ensure the common business processes which include the receiving and visualization of geospatial information.

The real situation is that many systems/applications, that carry out the communication with GIS objects, do not cooperate with each other and/or the services of different providers do not communicate with each other. When a client requires the data and the raster map about any GIS objects, the situation arises, when it is needed to invoke the Web services or their methods multiple times (for example, one Web service returns the streets set, that consists of raster map of streets and registered metadata of streets - two requests are generally needed, to get the total representation). This seriously overloads the work of GIS server and reduces the performance.

There is a new concept/solution of processing and visualization of GIS SOA requests that is proposed in this work. To avoid the overload of server with multiple requests to gain the exact set of geospatial data, the authors propose to create a special combined request to the GIS server data and to process/visualize the gained image at the client-side.

This paper is organized as follows. The view about GIS SOA is given in Chapter II. Chapter III is devoted to visual analysis of GIS SOA. The optimized data translation in GIS SOA and concatenated steganographic model appropriate for processing of geospatial data is described in Chapter IV. The last chapter is devoted to the conclusions of the article and the future directions of development and research.

\section{GIS SOA}

The Service Oriented Architecture (SOA) is a method of creating business applications that implement the common services for supporting business functions. The base component of the architecture is a service. It is possible to describe different business processes, mutual integration of which ensures the implementation of whole information system. The information about geographic objects is received from different, separate places and in different formats. In particular it concerns the outer environment, where the information is received from different sources and according to different data models and formats. The SOA approach can be used in the area of GIS. It ensures more elastic mechanism for visualization, control and distribution of GIS units. This mechanism is ensured by special geospatial Web services. The GIS SOA conceptual model is presented in Figure 1.

GIS is a proven and valuable technology, which plays an important role in the present SOA strategies and initiatives. By applying SOA, organizations can integrate GIS in their existing systems, to ensure the open access to the common geospatial data, services and applications. 


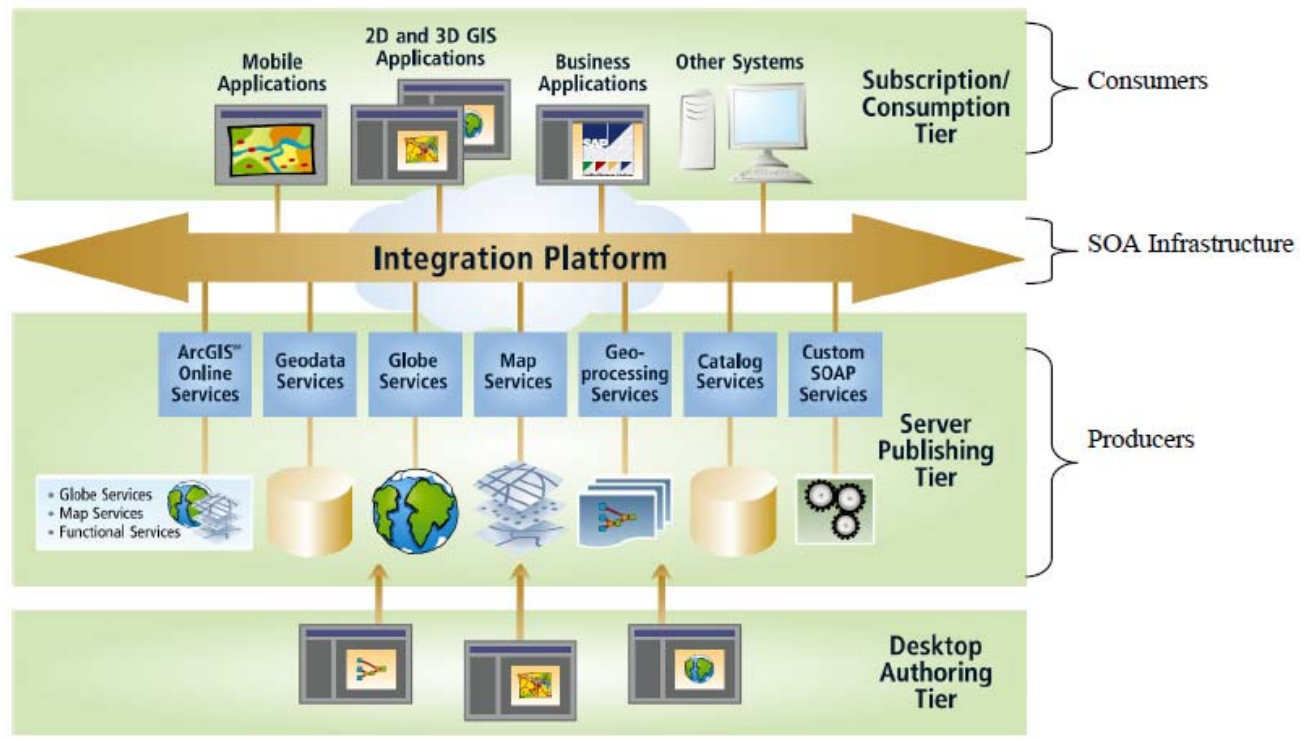

Fig. 1. GIS SOA conceptual model [1]

\section{a. GIS Web services}

GIS Web services can be grouped into three categories [2]:

- Data services: the services of such type are strongly associated with specific data sets and ensure the access to specified data parts. The WMS (Web Mapping Service), WFS (Web Feature Service), WCS (Web Coverage Service) and other services can be included in such category. The WMS returns the visual 2D maps with geospatial content. The WFS ensures the features of geospatial data, which are encoded in the GML language (Geography Markup Language). WCS ensures the access to non- visualized geospatial information.

- Services of process: the services of such type ensure the operations for data processing or transformation in such a kind that is defined with specified parameters of user. The services ensure such typical processing functions as projecting, coordinate transformation, rasterisation and vectorisation. The CPS (Coverage Portrayal Service), CTS (Coordinate Transformation Service) and other services might be included in this category.

- The services of register and catalogue: The services of such type ensure the possibility for users and applications to classify, register, describe, search, support and access the information about Web services. The FTHPIS (Fault Tolerant High Performance Information Service), Web Registry Service, Web Catalog Service and other services can be included in such category.

\section{b. The architecture of GIS Web services}

The fundamental components of GIS SOA architecture are:

- WMS reverts dynamically the spatial linked data maps, which can be gained from the geographical information. The service defines „map", which is the image of geographic information, and it is represented as a digital image file, that is appropriate for visualization on the computer screen. WMS ensures three methods:
1. GetCapabilities. The mandatory WMS method, that ensures the receiving of metadata about WMS service. This method describes all WMS service methods and their features.

2. GetMap. Mandatory WMS method, that enables obtaining of the map that can be shown on the screen. One of the most important request parameters is „LAYERS”. This parameter defines the geospatial objects for output (for example streets, rivers etc.). This method also possesses such important parameters as „STYLES” (defines how to output the result), „HEIGHT”, ,WIDTH”.

3. GetFeatureInfo. Optional WMS method, that ensures the receiving of additional features of WMS service. One of the most important request parameters of this method is "LAYERS". This parameter defines the geospatial objects, for which the supplementary information must be provided (for example - streets, rivers etc.).

- WFS defines the Web interfaces for access to geospatial data, that are based on features for accessing (vector data such as administrative information, streets etc.). WFS allows customer to get and renew the geospatial data which is encoded in GML language from different WFS services. The specification defines interfaces for data access and manipulation operations on geographical features, using HTTP as the distributed computing platform. The user of Web or service can collect, use and manage the geospatial data through these interfaces, for example, collecting the information about data representation from different places in the background mode, when WMS represents the map.

- WRS specifies the data of Web interfaces or services for searching in registers.

The fundamental components of service architecture of GIS Web are associated with SOA components. This is represented in Figure 2. 


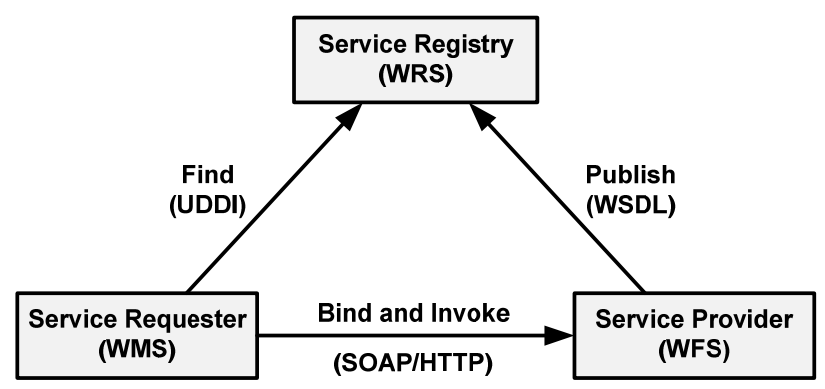

Fig. 2. GIS Web services representation with 3 major functionalities

In GIS context, the UDDI is metadata catalogue of registered services or server. Web services communicate by using SOAP protocol. This is for XML API functions that are provided with Web services. Each Web service contacts SOAP API via WSDL, ensuring with that the enclosing of functionality of simple services.

\section{III.THE VISUAL ANALYSIS IN THE FRAMEWORK OF GIS CLIENT}

One of the most important features of geographical information system (GIS) is the ability to ensure the analysis of geographical information being accumulated and its visual representation in the GIS client. Taking into consideration, that the major part of geographical information, by its nature, is the description of geometrical characteristics and relations of real world (for example - coordinates, sizes, etc.) that is supplemented with general attributes that have no analogues in the physical world, but which are important in the context of areas of human activity (for example - aspects of logistic: number of transportation transactions, the capacity of accumulation; economic indicators: profit, turnover; demographic indications: populations, density, etc.), the matter concerning the convenient combination of such kind of information from the visualization point of view becomes dominant.

To enable GIS client to define the request and represent the obtained data, there is a need for the following information attributes that are described in Table I:

TABLE I

THE SET OF ATTRIBUTES OF INFORMATION BEING VISUALIZED

\begin{tabular}{|c|l|l|l|}
\hline Nr. & $\begin{array}{l}\text { Information } \\
\text { unit }\end{array}$ & $\begin{array}{c}\text { Type of } \\
\text { information unit }\end{array}$ & \multicolumn{1}{c|}{ Description } \\
\hline \multicolumn{3}{|c|}{ Request parameters } \\
\hline 1 & $\begin{array}{l}\text { Desired data } \\
\text { display } \\
\text { mode }\end{array}$ & Mode ID & $\begin{array}{l}\text { Desired mode of work of GIS } \\
\text { client for performing the } \\
\text { processing of obtained data. }\end{array}$ \\
\hline 2 & $\begin{array}{l}\text { Request of } \\
\text { specified } \\
\text { parameters }\end{array}$ & $\begin{array}{l}\text { The parameters } \\
\text { of service call } \\
\text { (HTTP) }\end{array}$ & String. \\
\hline 3 & $\begin{array}{l}\text { Map } \\
\text { Response parameters }\end{array}$ & $\begin{array}{l}\text { Raster / vector } \\
\text { graphic image }\end{array}$ & $\begin{array}{l}\text { The map of geographic } \\
\text { region being visualized. }\end{array}$ \\
\hline 4 & $\begin{array}{l}\text { Data about } \\
\text { geographic } \\
\text { objects }\end{array}$ & $\begin{array}{l}\text { XML document } \\
\text { Array of strings / numbers, } \\
\text { which represents the } \\
\text { information for additional } \\
\text { characteristic of requested } \\
\text { objects. }\end{array}$ \\
\hline
\end{tabular}

The mode of common visualization session in the framework of GIS client is as follows:

- The definition of display mode parameters (the 1-st and the 2-nd information units) takes place in the user interface of GIS client;

- The call of WMS Web service takes place in order to acquire the resulting image (the 3-rd information unit);

- The call of WFS Web service (or the call of WMS service method GetFeatureInfo) takes place in order to obtain the remaining data set (4-th information unit);

- The visualization of received data and the work session within GIS client are being performed.

The main activities of GIS client can include the following:

- Actions that results in modification of geographical data (for example - dynamic creation of new routes, positioning of newly created objects in the map, etc.);

- Actions that do not change the data content (for example - navigating within the map / rotation / scaling of the image, etc.).

Taking into consideration that the returned map is a twodimensional image (while the attributes of geographical objects do not have the same restriction and these create the supplementary dimensions of information), the question concerning increase of number of visualization dimensions from classical two-dimensional space to three-dimensional space becomes important. This ensures more informative data placement. The specific application of visualization scenario and more detailed specification of involved data are presented below.

\section{a. The example of visual analysis}

The analysis of railway infrastructure of Latvia is used as an example, aiming to identify regions, where the increase of number of stations and routes is useful, regarding the aspects of demographic population in certain regions. The visualization mode in this case represents the map of Latvia with existing railway infrastructure (that consists of stations and connecting railways) together with the heightmap that represents relations between numbers of passengers and surrounding population in particular region. The topographic and demographic maps are obtained using the WMS service method GetMap, while calling the WMS method GetFeatureInfo provides the exact coordinates (geographical latitude / longitude), topology of stations / railways and the average amount of passengers handled (numerical value).

According to Table I, the content of information in this case is as follows (see Table II):

TABLE II

THE SET OF VISUALIZED INFORMATION ATTRIBUTES FOR ANALYSIS OF RAILWAYS OF LATVIA

\begin{tabular}{|c|l|c|}
\hline Nr. & $\begin{array}{c}\text { Information } \\
\text { unit }\end{array}$ & \multicolumn{1}{c|}{ Context } \\
\hline \multicolumn{2}{|c|}{ Request parameters } \\
\hline 1 & $\begin{array}{l}\text { Desired data } \\
\text { display mode }\end{array}$ & 3D_PROPERTIES_GRID \\
\hline 2 & $\begin{array}{l}\text { Request of } \\
\text { specified } \\
\text { parameters }\end{array}$ & Topographic_Map + Demographic_Map \\
\hline
\end{tabular}




\begin{tabular}{|c|c|c|}
\hline \multicolumn{3}{|r|}{ Response parameters } \\
\hline 3 & Map & \\
\hline 4 & $\begin{array}{l}\text { Data about } \\
\text { geographic } \\
\text { objects }\end{array}$ & $\begin{array}{l}\text { Stations: }= \\
<\text { S1: }\{100.23,78,89\} ; \text { S2: }\{98.56,12,45\} ; \ldots>; \\
\text { Railways:= } \\
<\text { S1-S2; S2-S3; S2-S4; S3-S4; S4-S5; ...; } \\
\text { Attributes:=<S1:78; S2:124; S3:46; ... }\end{array}$ \\
\hline
\end{tabular}

While performing the interpretation of this information using methods of spatial visual analysis, that rely on heightmaps and image processing filters for obtaining the interpolated attribute values (detailed information about this approach is presented in literature source [3]), it is possible to gain the following results (see Figure 3):

a)
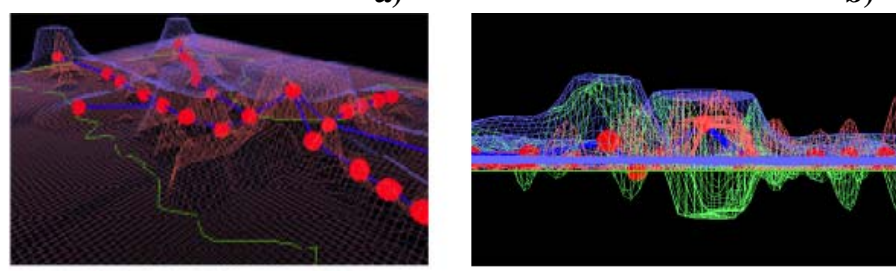

Fig. 3. The result of visualization of Latvia railways in the GIS framework

Image ,a)" provides the unified visual representation of \{Stations, Roads, Attributes\} data arrays (the capacity of existing Latvian railway infrastructure is marked with blue colour, the population in certain region - with red). In turn, the result in the image ,b)" enhances the result by providing supplementary visual heightmap (green) that represents the difference of these two data arrays in certain area of region and can be used for convenient identification of regions, where the expansion of the infrastructure is required.

According to this scenario, the data obtaining, while making request and waiting for answer from the server, requires more time (considering that multiple data load transactions are necessary). From the point of view of GIS visualization subsystem it would be more convenient to eliminate the delay of procedures of generating of the final image while waiting for an answer from WMS/WFS Web services. Instead, working with unified information source, which is obtained with the single WEB service, would be more convenient. For this purpose, the authors of this article offer to apply the steganographic implementation of information hiding.

\section{ThE Optimized ARCHITECTURE OF TRANSMISSION OF GEOSPATIAL DATA}

After digitalization geospatial data is separated in GIS server and, in turn, saved in 2 possible formats - raster and vector. The images of aero photography, maps, drawings of territorial division of land, etc. are classified as raster data. The vector format data, by its nature, represents the information that is already strictly structured and typified, for example, map metadata, measurements of cadastral units, names of streets, coordinates of objects etc. Statistically, every second request from the client is associated with the retrieval of map that, actually, requires data of both kinds to visualize, manipulate and correctly interpret real-world objects. So, our suggestion for optimization of this approach is based on idea that the mentioned information has to be provided to client simultaneously and in synchronized manner, so the supplementary GIS SOA service for calling of service method is not needed, resulting in acceleration of time-consuming data transmission through communication channel. The fact must be considered, that such optimization could not be complete, if two concurrently sent geospatial data sets enlarge the transmitted traffic. Therefore the additional requirement was introduced for the architecture being created, so it can ensure the biggest capacity of data transmission while keeping the same number of traffic bits. Additionally, the vector data capacity required from client (that specifies certain geospatial layer in the raster, for example, feature of objects, directions of movements, etc.) in comparison with the raster map itself is much lower.

Taking into consideration the aforementioned and the fact, that GIS SOA geospatial raster data is represented by the form of image via WMS service, the decision was made to adapt the technology that is able to ensure the hiding of textual data in the image without significant distortions of its content. The digital steganography was considered as such a technology which researches a set of methods for data hiding in the bit sequence (for example - within the text, digital images, audio or video streams). Nowadays the application sphere of steganographic methods and algorithms is privacy protection (for example, protecting of confidential information from unauthorized access, suppression of surveillance system and the espionage network resource management, copyright protection), but solutions exist, in which the application of steganography is oriented to the significant feature of data hiding - constant number of bits transmitted at the increased amount of data [4]. More information about the essence of steganography and the conceptual design of steganographic systems can be found in the original research articles $[5,6,7]$.

According to the fact, that the synchronization of geospatial layers is also needed, the concatenated steganographic model (its design is available in the section A of Chapter IV), the base concept of which is described in the literature source [4], adapted to GIS layers is located at the core of the developed architecture (see Figure 4)

The embedding procedure of concatenated steganography becomes an integral part of WMS service and it is activated before sending WMS response, in order to put the raster data of geospatial layers and appropriate metadata required by client application. In other words, receiving the request, the WMS service performs such steps:

1. The raster maps of required layers are selected according to request parameters (actually this is the result of GetMap method of simple WMS service) in the GIS server database, together with vector data, that, in turn, describes the features of visible objects of each layer and/or attributes of these (essentially it is the result of execution of simple WMS service method DescribeFeatureInfo or the resulting data set of WFS service). 


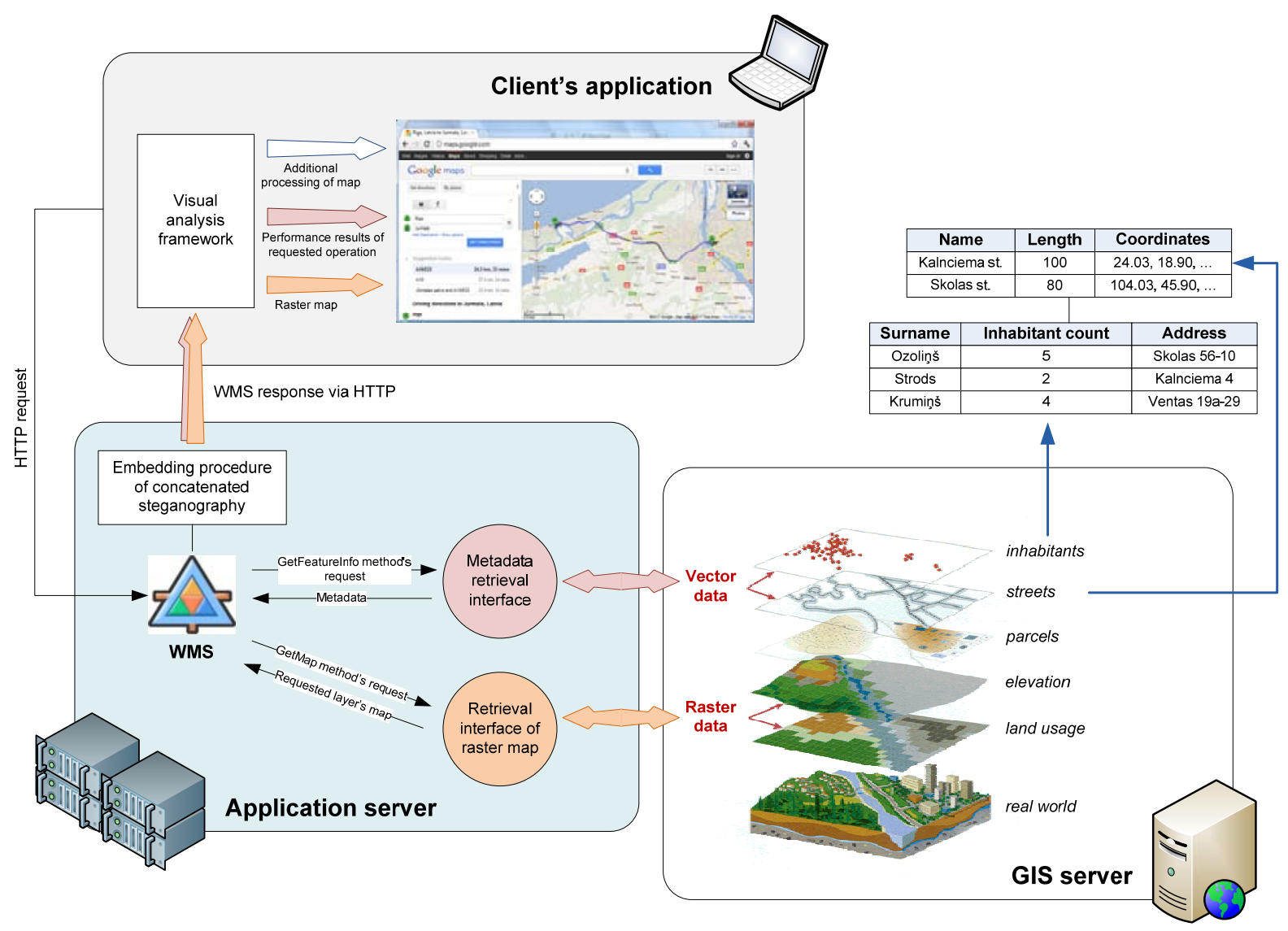

Fig. 4. The data transmission in GIS SOA adapted to visual analysis

2. By using the concatenated steganographic model:

- Each selected layer is supplemented with corresponding metadata or objects characteristics;

- The included raster maps of layer are arranged according to size in ascending order;

- Each small-sized raster map is embedded into the layer of map of larger size - this process continues iteratively, while the creation of a single raster is not completed.

3. The resulting raster image is returned via active HTTP connection.

The result of WMS service execution is transferred to client's tool for visual analysis that is responsible for retrieving of layer with metadata and preprocessing of data being visualized, for example, calculation of optimal route or creating of three-dimensional map image. After all, the geospatial map and the result of its additional processing are presented to the customer (depending on functionality being ensured by the visualization tool), while only ONE data transmission from GIS server is required.

The proposed architecture of data transmission is fully compatible with current GIS SOA architecture. This means that WMS service of expanded functionality that implements the embedding procedure of concatenated steganography, works according to generally accepted OGC standards and supports both POST and GET requests. In order to support our solution, the list of WMS service available parameters (available at http://www.opengeospatial.org/standards/wms) must be expanded with new values that will determine the operation mode of the service. The newly attached request parameters depend on tasks, solution of which is required by application of this service (one of such application examples was examined in the Chapter III). So, in case, if the used software is developed with support of previous WMS service, where these parameters were not specified, the returned raster map will be reflected in standard mode without extracting of metadata and its additional processing. Otherwise, additional functionality of visualization and processing of geospatial data will be available on client's tool.

\section{. Concatenated steganographic application in GIS context}

The concept of concatenated steganographic model (CSM) is represented in the source [4] and it is based on the idea concerning the multiple use of the procedure of data embedding before transmission. So the CSM is able to ensure enhanced security of transmission of the hidden information, as well as to implement the synchronization mechanism of bits sequence, that is important regarding the distribution of geospatial data content. As it was already mentioned, the distribution of geospatial data content requires both the transmission of raster map and providing the structured metadata of suitable objects. So it is possible to conclude, that the necessity exists regarding the transmission of textual symbols and image bit streams. Taking into consideration the fact, that the metadata layer has more interpretative meaning, its total amount in comparison with the size of raster layer is much smaller. Thereby the metadata will be embedded into 
raster and not vice versa. In addition, it should be noted, that the multi-layer data is requested within GIS SOA.

In order to ensure everything mentioned above, CSM concept was improved and adapted to data being kept within the GIS system (see Figure 5). As it can be seen, that the resulting stego-media container (that represents the layered raster map combined with its metadata) in one coder's session becomes the input parameter for the next coder. Additionally, the input procedure of each level intends the use of kind of steganographic methods that is based on redundancy of visual information (image steganography), which means, that the hiding of bits always happens in the image, for example:

- Hiding procedure of the 1-st layer of geospatial data in its raster map is taken place on the level 0 , but

- The newly created stego-media object in the $1^{\text {st }}$ level (map + metadata) will already be hidden together with the metadata of the $2^{\text {nd }}$ geospatial layer in the raster map of $2^{\text {nd }}$ layer.

Considering that the result of the activity of previous level is directly applied in the next level, it is possible to conclude, that the relationship [1-1] exists between layer data embedded between $\mathrm{N}-$-level and $\mathrm{N}+1$ level data container. In other words, the bit stream of one layer is synchronized with the bit stream of another layer, maintaining the GIS data integrity and consistency.

It should be noted, that the volume of necessary data container (equals to raster map of geospatial layer) increases significantly in each subsequent level. That is why before starting embedding process in each CSM level it is meaningful to select such map from the existed layer maps, that will be able to embed both the stego-media data of previous level, as well as current level. In case if it is not possible to arrange and find the most appropriate data container for steganographic embedding, then it happens according to size (the calculation method is described in the source [4]) operation of welding of small size raster maps (together with combining of according layer metadata), until the required data container is obtained. And only after that the embedding process continues.

In order to check the possibility of application of image steganography in the GIS systems and CSM correct interpretation in GIS context, the experiments were performed with Old Riga geospatial data. The layers of this data include (the metadata of certain layer are shown in the brackets that additionally specifies the raster map of this layer):

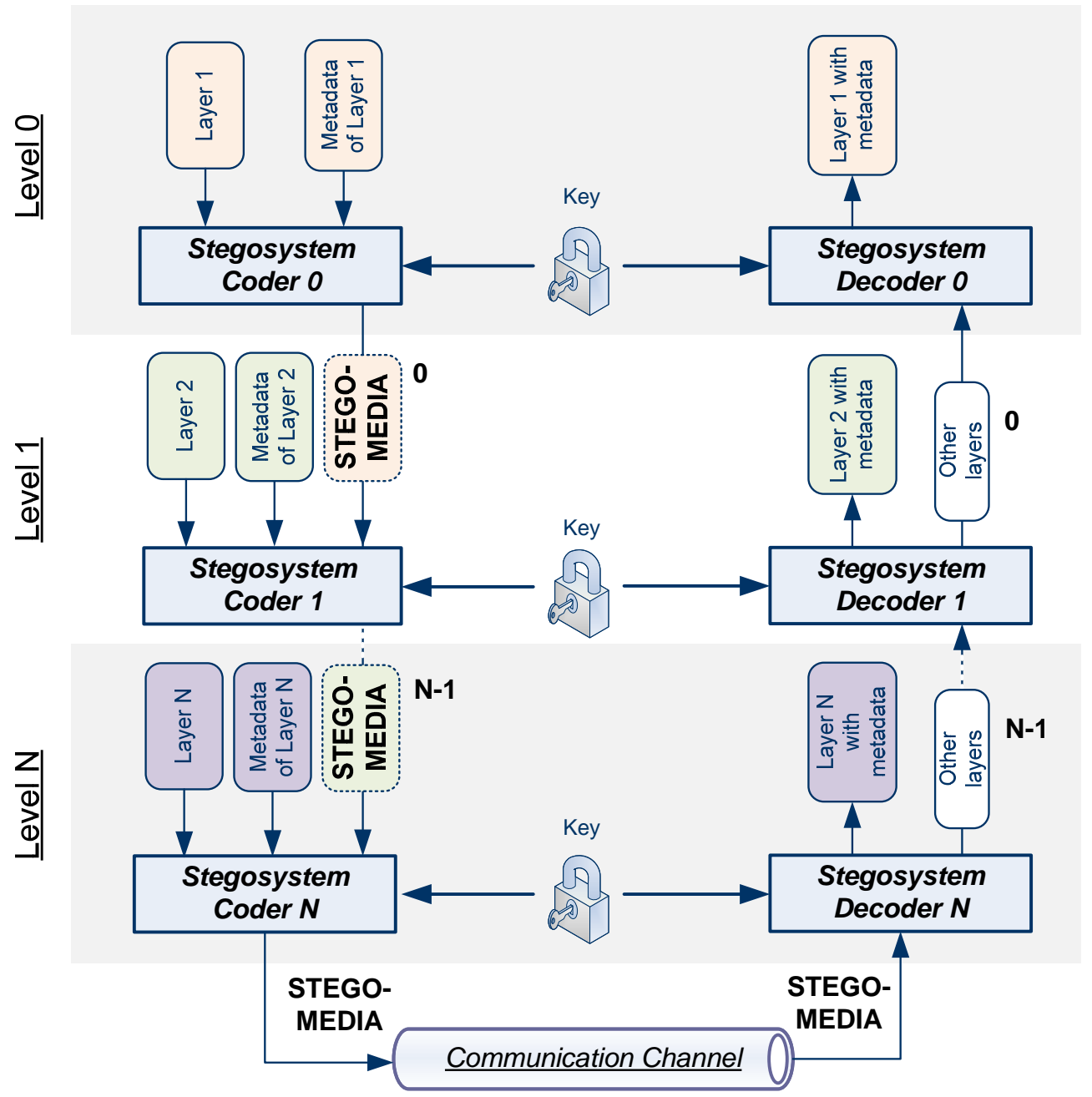

Fig. 5. Concatenated steganographic model appropriate for GIS 
- Buildings (number of stores, structured addresses, objects of building shapes);

- Streets (coordinate corteges, names);

- Monuments, memorials (names of the most important places, coordinates, year of establishment).

The popular steganographic method LSB (Least Significant Bits) was applied to embed the indicated data into the layer map. LSB method performs insignificantly colour changes in the image, which, in turn, raise the brightness changes that are invisible to human's eye [5]. If one bit is used for one message embedded in each byte, then it is possible to embed one message symbol in eight container bytes. But the LSB method can be applied for different number of bytes, while changing multiple bits in a byte. And specifically this feature was used for GIS layers. The obtained results proved that the simultaneous change of the smallest 4 bits of each byte won't create the distortion of raster map content. In other words, using LSB method for any GIS layer, valuable information could be embedded in the raster map, which volume takes up to $50 \%$ of map size. Considering that the raster map takes 400 $\mathrm{KB}$ in average, the built-in metadata capacity is equal to 200 $\mathrm{KB}$ that is enough.

\section{RELATED WORKS}

Taking into consideration that GIS domain continue development and according solutions became integral parts of tools for support of strategic decision making, nowadays many products are being developed, allowing to solve standard tasks in the context of processing of geographic information.

Although the number of such products and its diversity does not allow for full review, the information regarding the different generally accepted and widely used end user visualization solutions (Desktop GIS clients) is given within this article:

- GRASS GIS - open source software that was developed since 1982 and gradually evolved into powerful package, which enables not only processing of geographic data / planning of environment development, but also allows for scientific research of data. This system enables to perform raster and vector graphics processing, and additionally, ensures the classic techniques of image processing [8]. The data saving and retrieving occurs with dBase II DBVS, but other SQL-based solutions are also possible (for instance - MySQL, PostgreSQL/PostGIS, SQLite) while ensuring the condition that data is kept locally. This means that GRASS does not provide direct support of the architecture advantages of Web services and additional integration works are needed to ensure appropriate functionality [9].

- Quantum GIS - open source software which is being developed since 2002. It includes the possibility of viewing, modifying and analysis of geographic data [10]. While aiming for expansion of main functionality the maintaining of dynamically connected modules (plugins) is supported (these can be created with $\mathrm{C}++/$ Python). The data retrieving is performed with SQLite / GDAL /
PostGIS / PostgreSQL, but the service also supports WMS/WFS according to OGC specification.

- ILWIS (Integrated Land and Water Information System) is a GIS client, which supports not only general data processing, but also is capable of automated creation of maps, while performing the digitalization of data input [11]. Until 2007 it was a commercial product with emphasis on managing of water resources, but later it was switched to open source licensing model. This tool features both classical two-dimensional visualization and the ability to display three-dimensional maps. The $\mathrm{ADO} / \mathrm{ODBC}$ were used as the main mechanisms of data retrieving, but the initial implementation of WMS-type service occurred in the last product version 3.7.

- $u$ Dig - open source software that is being developed on the base of „Eclipse" platform using programming language Java. The last version is able to cooperate with other GIS solutions (for example - using abovementioned GRASS tool for complicated operations with vector data). Additionally it supports saving/retrieving of geographical data of several levels (using PostGIS / textual files for description of geographic data) and according visualization [12]. The integrated support for WMS, WFS, WCS family is embedded in uDig, as well as supplementary standards GeoRSS and KML.

- gvSIG - the open-source solution which is based on Java. The last version 1.11 allows displaying and processing data of several files (for example, raster BMP, GIF, TIFF, JPEG, JP2, PNG, VRT, ERDAS, PIX, AUX, ADF, MPR, MPL, ASC, PGM, PPM, RST, RMF, NOS, KAP, HDR, RAW, etc.; vector - SHP, DXF, GML, DWG, DGN, KML, etc.) with intention to allow the user to manage the data effectively and solve the complicated management and planning problems in the domain of geographic resources [13]. The access to the locally kept data is ensured through several mechanisms PostGIS, MySQL, ArcSDE, Oracle, JDBC, CSV, while it is possible to use OGC standard for distant access (WMS, WFS, WCS, WFS-T, WPS), as well as ArcIMS service.

The summarized comparison of mentioned solutions and the new proposed approach is presented in Table III.

As it can be perceived from the table, the proposed architecture supports functionality of all existing solutions, while simultaneously offering unique feature to optimize the data transmission procedures without changes in logic of existing infrastructure.

\section{CONCLUSIONS}

The basic principles of creating GIS SOA architecture are reviewed in this article while discovering the problems of inefficient data transmission for GIS visualization solutions on the client side. This problem is associated with the fact, that from the view of visualization in the framework of GIS client, it is not important how the data is transferred to the graphical system. Therefore the number of sent requests at the GIS server is doubled in existing GIS SOA solutions, because the 
TABLE III

COMPARISON OF SOLUTIONS

\begin{tabular}{|c|c|c|c|c|c|c|}
\hline Ensured functionality & $\begin{array}{l}\text { GRASS } \\
\text { GIS }\end{array}$ & $\begin{array}{l}\text { Quantum } \\
\text { GIS }\end{array}$ & ILWIS & uDig & gvSIG & $\begin{array}{l}\text { Optimized } \\
\text { architecture }\end{array}$ \\
\hline Usage of raster and vector graphics & $\nabla$ & $\nabla$ & $\nabla$ & $\nabla$ & $\nabla$ & $\nabla$ \\
\hline $\begin{array}{l}\text { Availability of different geospatial } \\
\text { data layers }\end{array}$ & $\nabla$ & $\nabla$ & $\square$ & $\square$ & $\nabla$ & $\square$ \\
\hline Three-dimensional data visualization & $\nabla$ & & $\nabla$ & & $\square$ & $\square$ \\
\hline $\begin{array}{l}\text { Support of loaded data modification } \\
\text { and preprocessing }\end{array}$ & $\square$ & $\nabla$ & $\nabla$ & $\square$ & $\nabla$ & $\nabla$ \\
\hline Support of decision making processes & $\nabla$ & $\nabla$ & $\nabla$ & $\nabla$ & $\nabla$ & $\nabla$ \\
\hline Service-oriented data retrieval & & $\nabla$ & & $\square$ & $\nabla$ & $\square$ \\
\hline Platform independence & $\nabla$ & $\square$ & & $\square$ & $\square$ & $\square$ \\
\hline Unified approach to data retrieval & & & & & & $\square$ \\
\hline
\end{tabular}

raster and vector data are received separately, although these are needed simultaneously for full-fledged work on client side. Therefore, the attempt was made to solve this problem on the business level within this work, by synchronizing the geospatial flows of raster and vector before they get into the customer application. For this purpose, achievements of embedding of digital steganography data in the bits flows are applied, which results in the adaptation of so called concatenated steganographic model to the data kept within the GIS system. Additionally, the sample of three-dimensional application of computer graphic was developed, that allows optimizing of the visual placement of data, using one reserve dimension for the displaying of supplementary attributes in comparison with classical "flat" displaying.

The implementation confirmed ability of our approach to enable successful data analysis within GIS client, based on unified retrieving of geospatial information. As described in Chapters III and IV, appropriate use case allowed identifying of potential improvements of railway infrastructure of Latvia which is both reliable (due to the fact that it is based on automated visual analysis of existing capacity of the infrastructure and demographic characteristics) and effective in terms of usage of geospatial Web services (due to the introduction of CSM concept which allowed to perform mentioned analysis within just one session of data retrieval). In fact, all mentioned useful conclusions were obtained while providing only limited set of parameters (3D_PROPERTIES_GRID / Topographic_Map + Demographic_Map) for one-time calling of a single Web service within the GIS client.

A set of distinctive advantages of the proposed solution is as follows:

- Ability to obtain comprehensive results of visual analysis while providing well-chosen and relatively small set of parameters for specification of desired data within single call of geospatial Web service.

- Provided architecture of data transmission is fully compatible with any of existing GIS SOA solutions, where WMS and WFS services are used.

- The dependence on steganographic hiding techniques allows organizing new kind of licensing models (like copyrights) in geospatial data infrastructure.

A set of known additional limitations is as follows:
- A necessity to perform additional calculation within GIS client before the visualization session can start, in order to extract the encoded data with properties of geographical objects from the image of the map.

- Additional processing time is needed for WMS service before sending the response in order to embed metadata into the raster data of the layer. So totally it can influence the execution time of single HTTP request.

The potential further research development directions are as follows:

- Analysis of standard GIS application scenario aiming to define the parameters of necessary modes with metadata transmission format.

- Standardization of information formats being contributed according to adapted CSM geo-data.

- Application of universal stegoconstructor [14] in CSM, to find the most appropriate stegonographic model for geospatial data media, as well as guarantee the stability of placed metadata against transformations of different kind during the transmission sessions within the SOA architecture.

\section{REFERENCES}

[1] An Esri, Geospatial Service-Oriented Architecture http://www.esri.com/library/whitepapers/pdfs/geospatial-soa.pdf [Accessed: Sept. 16, 2011]

[2] Sahin K., Gumusay M. U., SERVICE ORIENTED ARCHITECTURE (SOA) BASED WEB SERVICES FOR GEOGRAPHIC INFORMATION SYSTEMS, Istanbul, Turkey, http://www.isprs.org/proceedings/XXXVII/congress/2 pdf/5 WG-II5/03.pdf [Accessed: Sept. 16, 2011]

[3] V. Zabiniako and P. Rusakov, Combined Representation of Data with Graphs and Heightmaps for Visual Analysis and Spatial Decision Support. Proceedings of the IADIS International Conferences. Computer Graphics, Visualization, Computer Vision and Image Processing 2010. July 27-29, 2010. Germany: IADIS Press, pp. $184-192$.

[4] Yershov A. Concatenated Steganography as an Alternative Solution for Data Streams Synchronization. Local Proceedings of 13th EastEuropean Conference (ADBIS 2009). Associated Workshops and Doctoral Consortium. September 7-10, 2009. Latvia: Riga, pp. 444-452.

[5] Yershov A., Rusakov P. The Comparative Analysis of Digital Steganographical Methods LSB and Kutter // The 46th Scientific Conference of Riga Technical University, Computer Science, Applied Computer Systems - October 13-14 - Riga, Latvia - 2005 - published in Scientific Proceedings of Riga Technical University, 5th series, Computer Science, Applied Computer Systems, Vol. 26, RTU Publishing, RTU - 2006 - pp. 186-197. 
$2012 / 13$

[6] Eric Cole, Ronald D. Kruz. Steganography Art of Covert Communication. Indianapolis: Wiley Publishing, 2003, 361 pages.

[7] Ingemar K. Cox, Matthew L. Miller, Jeffrey A. Bloom, Jessica Fridrich, Ton Kalker. Digital Watermarking and Steganography. Burlington: Morgan Kaufmann Publishers, 2009, pp. 46-56, 469-494.

[8] P. Dunlavey, "GRASS GIS: A Useful Tool for the Mountain Cartographer", International Cartographic Association (ICA) 2002 Mountain Cartography Workshop, Timberline Lodge, Mt. Hood, Oregon, 2002. [Online]. Available: http://www.mountaincartography.org/mt_hood/pdfs/dunlavey1.pdf [Accessed: Sept. 16, 2011].

[9] W. Bergenheim, L. Sarjakoski and T. Sarjakoski, "A Web Processing Service for GRASS GIS to Provide on-line Generalisation", 12th AGILE International Conference on Geographic Information Science 2009, Leibniz Universitat Hannover, Germany. [Online]. Available: http://www.ikg.uni-hannover.de/agile/fileadmin/agile/paper/126.pdf [Accessed: Sept. 24, 2011].

[10] "Quantum GIS: User, Installation and Coding Guide", Quantum GIS Development Team, 2011. [Online]. Available: http://download.osgeo.org/qgis/doc/manual/qgis-.7.0_user_guide_en.pdf [Accessed: Oct. 1, 2011].

[11] "ILWIS 3.0 Academic User's Guide", International Institute for Aerospace Survey and Earth Sciences (ITC), Enschede, The Netherlands, May 2001. [Online]. Available: http://www.megwrm.aun.edu.eg/sub/workshop5/pdf/ilwis user_guide30 .pdf [Accessed: Aug. 14, 2011].

[12] G. Watry, "Introduction to User-Friendly Desktop Internet GIS", Center for Ocean-Atmospheric Prediction Studies, 2007.[Online]. Available: http://gis.coaps.fsu.edu/FOSS GIS/Introduction to uDig 1110 RC8.p df [Accessed: Sept. 18, 2011].

[13] S. Lopez, "Introduction to gvSIG as desktop GIS/SDI client", First Open Source GIS UK Conference - University of Nottingham, June 2009. [Online]. Available: http://gvsigdesktop.forge.osor.eu/downloads/pub/documents/learning/gvsigcourses/gvsig_des_1.1.x_u_en/gvSIG_workshop_OSGIS09.pdf [Accessed: Sept. 24, 2011].
[14] Yershov A., Rusakov P. Kutter Steganographical Method's Improvement and Concept of Universal Stegoconstructor. Scientific Journal of RTU. 5. series, Datorzinātne. - 38. vol. (2009), pp 198-208.

Andrew Yershov was born in 1984 and currently is a PhD student at Riga Technical University at the Faculty of Information Technology and Computer Science. Mg.sc.ing. (2008), B. sc. ing. (2005) - Riga Technical University (RTU), Institute of Applied Computer Systems.

IT project manager in the Latvian company "ABC software". Field of interests: computer science, data integration. Special interests: programming paradigms, distributed systems, Web technologies, steganography and steganalysis, cross-platform development, signal processing.

Vitaly Zabiniako was born in Riga, Latvia in 1983 and currently is a $\mathrm{PhD}$ student at Riga Technical University at the Faculty of Information Technology and Computer Science. Master's degree in computer science at Riga Technical University, 2007.

Systems analyst in private IT company "ABC software", previously system administrator at Riga Technical University, Institute of Applied Computer Systems. Example of previous publications: V. Zabiniako, P. Rusakov. Comparative Analysis of Visualization Aspects in Technologies Direct3D and OpenGL. Scientific Proceedings of Riga Technical University, Computer Science, series 5, vol. 26, pp 209-221 (2006). Interests and dissertation are connected with computer graphics and information visualization.

Awards - diplomas with distinction (Bachelor of engineering science in computer control and computer science; Master of engineering science in computer systems).

Pavel Semenchuk was born in Riga, Latvia in 1984 and currently is a PhD student at Riga Technical University at the Faculty of Information Technology and Computer Science. Master's degree in computer science at Riga Technical University, 2008.

IT project designer/programmer in company "ABC software". Field of interests: computer science. Special interests: SOA, programming paradigms, distributed systems, Web technologies. 